\title{
Evaluation of 3D-CPA, HR-HPV, and TCT joint detection on cervical disease screening
}

\author{
HUI LIANG, MIN FU, JIAN ZHOU and LEI SONG

\begin{abstract}
Maternal and Child Care Service Center of Xuzhou, Xuzhou Maternal and Child Care Service Center of
\end{abstract} \\ Xuzhou Medical College, Xuzhou, Jiangsu 221009, P.R. China \\ Received December 23, 2015; Accepted June 2, 2016
}

DOI: $10.3892 / \mathrm{ol} .2016 .4677$

\begin{abstract}
The application value of three-dimensional color power angiography (3D-CPA), high-risk human papillomavirus (HR-HPV), ThinPrep cytology test (TCT) joint detection on cervical disease screening was investigated. In total, 1,900 patients that were examined in Gynecological and Cervix Clinic of Maternal and Child Care Service Center of Xuzhou from June 2012 to March 2015 were enrolled in the present study. After admission, the patients underwent TCT, HR-HPV and 3D-CPA examinations, and vascular morphology and typing, vascularization index (VI) were recorded. Colposcopic biopsy was performed in patients with a positive outcome of any of the three indices. Pathological diagnosis was taken as the golden standard to assess the sensitivity, specificity, diagnostic rate, and Youden index of the three methods being used independently or jointly. Of the 1,900 patients, 276 cases (14.53\%) were HR-HPV-positive, 214 cases $(11.26 \%)$ were VI-positive and 164 cases $(8.63 \%)$ were TCT-positive. A total of 418 cases were confirmed with a positive outcome of any of the three indices and a cervical biopsy was obtained. Of the 418 cases, 162 cases (38.75\%) were diagnosed with chronic cervicitis, 146 cases with low-level cervical intraepithelial neoplasia (CIN) (34.93\%), 104 cases $(24.88 \%)$ with high level CIN, 6 cases $(1.44 \%)$ with cervical cancer. Histology more than low level CIN was defined as positive: i) screening results when the three methods were used independently: HPV was confirmed with the highest sensitivity $(90.63 \%)$, VI with the highest specificity (83.95\%), and HPV with the highest diagnostic accuracy (83.73\%); ii) screening results under HPV+TCT and HPV+TCT+VI: HPV+TCT+VI was confirmed with the highest sensitivity and specificity: sensitivity (94.53\%), specificity $(81.48 \%)$, diagnosis coincidence rate $(89.47 \%)$ and the highest Youden index of 0.760; and iii) vascular morphology
\end{abstract}

Correspondence to: Dr Min Fu, Maternal and Child Care Service Center of Xuzhou, Xuzhou Maternal and Child Care Service Center of Xuzhou Medical College, Xuzhou, Jiangsu 221009, P.R. China E-mail: dme225@163.com

Key words: three-dimensional color power angiography, human papillomavirus, ThinPrep cytology test, cervical lesions, screening and grading were significantly different in the early stage cervical carcinoma, high level CIM, and cervicitis groups. In conclusion, the joint detection of 3D-CPA, HR-HPV, and TCT improved the sensitivity and accuracy of cervical disease screening. 3D-CPA technology may therefore be used as an auxiliary screening method for cervical cancer.

\section{Introduction}

Cervical cancer is the leading cause of mortality in women, with the highest mortality of all gynecologic tumors (1). World Health Organisation (WHO) has admitted that as many as 529,409 women worldwide are likely to be diagnosed with cervical cancer, and 274,883 are likely to succumb to the disease. No less than $80 \%$ of this burden is borne in less developed countries (1). In America, it is estimated that there are approximately 92,136 cases and 37,640 mortalities annually, representing a significant economic loss, projected at being potentially greater than US $\$ 3,6$ billion (2). It takes approximately 5-10 years for cervical intraepithelial neoplasia (CIN) to develop into cervical cancer and the progression of disease has reversibility. If CIN is screened out and treated timely and completely, a lesion may disappear or develop into cancer (3). Therefore, the key for solving this problem lies in effective screening, which is also the best way to prevent and control cervical cancer to a large extent. The sensitivity of ThinPrep cytology test (TCT) detection is only $55-80 \%$ and HPV, although has the highest sensitivity, has very poor specificity. Thus, a safe, effective and joint detection method for the early diagnosis of cervical cancer should be identified. Three-dimensional (3D) color power Doppler may provide an invasive, real-time, objective and overall assessment on the blood supply condition of patients, thus, it is an ideal technology for the evaluation of the blood supply of cervical lesions (4). In this investigation, we have taken pathology diagnosis as the golden standard to determine the application value of three-dimensional color power angiography (3D-CPA), high-risk human papillomavirus (HR-HPV), and TCT joint detection.

\section{Patients and methods}

Patients. A total of 1,900 patients that were examined at the Gynecological and Cervix Clinic of Maternal and Child Care Service Center of Xuzhou (Jiangsu, China) from June 2012 to 
March 2015 were enrolled in our study. The patients were aged 20-65 years, with an average of $35.5 \pm 4.6$ years. The inclusion criteria for the present study was patients without history of medical application on vagina, cervical precancerous lesions or conization of cervix in the previous week. Patients in the menstrual or gestation period were excluded. TCT, HR-HPV and 3D-CPA examinations were performed and vascular morphology and typing, vascularization index (VI) were recorded. Colposcopy biopsy was performed in patients with a positive outcome of any of the three indices. Pathological diagnosis was taken as the golden standard for assessment. The patients enrolled were required to fill a case report form and provide written informed consent. The study was approved by the Ethics Committee of Maternal and Child Care Service Center of Xuzhou.

Sample collection. Cells were collected from the cervical canal via TCT, and HPV cervix brush, and the brush was placed into preserving fluid for examination. The collected samples were preserved at $4^{\circ} \mathrm{C}$ for $\leq 3$ days. Abnormal secretion of menstruation and vagina was avoided while drawing samples.

Instruments. GE Voluson E8 color Doppler ultrasonic diagnostic apparatus (General Electric, Schenectady, NY, USA), intracavitary probe, 5-9 $\mathrm{MHz}$ was applied. Virtual organ computer-aided analysis (VOCAL) software was utilized to measure relevant vascular parameter values. Pulse repetition frequency (PRF) of 0.9 was selected, three-dimensional quantification sampling was set at high quality, a section was selected every $15^{\circ} \mathrm{C}$, and a total of 12 sections was selected from each lesion. Envelope path of the lesions were drawn manually.

$3 D$ power Doppler ultrasonography examination. First, we performed regular transvaginal 2D ultrasound examination on cervix under the grayscale mode and closely examined the properties of tumor in patients with lesions. For the patients diagnosed with obvious tumor in the cervix by ultrasonic examination, the tumor was taken as the region of interest. For the patients diagnosed without tumor in the cervix, the whole cervix was taken as the region of interest and screening was performed. Subsequently, the examination was switched to color power Doppler mode and 3D reconstruction was performed on the region of interest, 3D distribution and branch of cervical vessels were exhibited and typing of the vessels was observed. The typing standard for vessels under ultrasonography (1) included: type I vessels, vessels that were uniformly distributed, thin, and straight; type II vessels, vessels that had annular thickening locally; type III vessel, vessels that were disorderly and unsystematic locally and had a large number of branches; and type IV vessel, vessels that were non-uniform, distortedly clustered in the shape of a fire ball.

Manual outlining sampling method and 3D power Doppler histogram were used for quantitative analysis on the vessels and blood flow volume. The parameters obtained were: VI measured the number of color information in the region of interest and showed the number of vessels detected inside tissues, and examination on each patient was completed by the same sonographer using the same diasonograph and the same environment. A VI cut-off value of $\geq 3$ was defined as positive.
HPV-DNA detection and diagnostic criteria. HPV typing method - Hybribio medical nucleic acid molecule hybridization technique and its reagents (introduced from HybriBio, Hong Kong, China) were applied to typing and detect the 21 common HPV genotypes including 15 types of HR-HPV $(16,18,31,33,35,39,45,51,52,53,56,58,59,66$ and 68) and 6 types of low-risk types $(6,11,42,43,44$ and 8304). A positive result of any of the high-risk types was viewed as positive.

Multiple cervix punch biopsy under colposcope and endocervical canal curettage (ECC). Colposcopic biopsy was performed in patients with a positive outcome of any of the above three indices. For the patients that had abnormal lesions, samples were collected from the site of the lesion. For the patients that had no abnormal lesion, the samples were collected from sites 3, 6, 9 and 12 in the transformation zone. Furthermore, ECC was performed and the biopsy tissues sent for pathological examination. According to the degree of lesion, the lesions in chronic cervicitis were divided into low level CIN (CINI), high level CIN (CINII-CINIII glandular involvement), and cervical cancer. Histology of more than low level CIN was defined as positive.

Statistical analysis. SPSS 17.0 software (SPSS Inc., Chicago, USA) was applied for statistical analysis. Based on the $\chi^{2}$ test and the golden standard of pathology, the sensitivity, specificity, diagnostic rate and Youden index of the three indices under independent or united screening were computed.

\section{Results}

HR-HPV, TCT and 3D-CPA. The VI detection results showed that of the 1,900 patients, 276 cases $(14.53 \%)$ were confirmed HR-HPV-positive, 214 cases (11.26\%) VI-positive, and 164 cases $(8.63 \%)$ TCT-positive.

Results of biopsy under colposcopic and pathologic biopsy. A total of 418 cases were confirmed with a positive outcome of any of the three indices and a cervical biopsy was obtained. Of these, 162 cases $(38.75 \%)$ were diagnosed with chronic cervicitis, 146 cases with low-level CIN (34.93\%), 104 cases (24.88\%) with high level CIN, and 6 cases $(1.44 \%)$ with cervical cancer. Histology of more than low level CIN was defined as positive.

Comparison on the positive rate and pathological coincidence rate of HR-HPV and TCT. Of the 1,900 cases, 276 cases were confirmed with high-risk HPV infection, the positive rate being $14.52 \%$ and pathological coincidence rate $37.68 \%$. However, the HR-HPV-positive rate in high level CIN or above was $95.45 \%$ (105/110). Cases (164) were confirmed as TCT-positive, at $8.63 \%$ and the pathological coincidence rate was $82.93 \%(136 / 164)$ (Table I).

Pathological diagnosis was taken as the golden standard for the sole and joint assessment of the sensitivity, specificity, diagnostic rate, Youden index of HPV, TCT and VI on lesions more than CINI (Table II).

Cervical ultrasonography and vascular typing. Results from the chronic cervicitis and CINI groups indicated that the 
Table I. Comparison of the positive rate and pathological coincidence rate between TCT and HPV.

\begin{tabular}{|c|c|c|c|c|c|c|}
\hline \multirow[b]{2}{*}{ TCT cases } & \multirow[b]{2}{*}{$\mathrm{N}$} & \multicolumn{4}{|c|}{ Pathological results } & \multirow{2}{*}{$\begin{array}{c}\text { Pathological coincidence rate } \\
\text { with } \geq \text { high level CIN, n/total (\% }\end{array}$} \\
\hline & & Inflammation & CINI & High level CIN & $\mathrm{Ca}$ & \\
\hline ASCUS & 102 & 26 & 33 & 42 & 1 & $42 / 102(41.18)$ \\
\hline LSIL & 26 & 2 & 9 & 14 & 1 & $14 / 26(53.85)$ \\
\hline HSIL & 35 & 0 & 4 & 28 & 3 & 28/35 (80.00) \\
\hline SCC & 1 & 0 & 0 & 0 & 1 & \\
\hline High risk HPV & 276 & 44 & 128 & 98 & 6 & $98 / 276(35.51)$ \\
\hline
\end{tabular}

TCT, ThinPrep cytology test; HPV, human papillomavirus; CIN, cervical intraepithelial neoplasia.

Table II. Diagnostic efficiency of sole and joint screening of the three indexes on lesions more than CINI.

\begin{tabular}{lcccc}
\hline Index & Sensitivity & Specificity & Diagnostic coincidence rate & Youden index \\
\hline VI & $73.44 \%(188 / 256)$ & $83.95 \%(136 / 162)$ & $77.51 \%(324 / 418)$ & 0.574 \\
HPV & $90.63 \%(232 / 256)$ & $72.84 \%(118 / 162)$ & $83.73 \%(350 / 418)$ & 0.635 \\
TCT & $53.13 \%(136 / 256)$ & $79.01 \%(134 / 162)$ & $63.16 \%(264 / 418)$ & 0.321 \\
HPV+TCT & $92.19 \%(236 / 256)$ & $76.54 \%(124 / 162)$ & $86.12 \%(360 / 418)$ & 0.687 \\
HPV+TCT+VI & $94.53 \%(242 / 256)$ & $81.48 \%(132 / 162)$ & $89.47 \%(374 / 418)$ & 0.760 \\
\hline
\end{tabular}

CIN, cervical intraepithelial neoplasia; TCT, ThinPrep cytology test; HPV, human papillomavirus.

Table III. Vascular typing characteristics of cervical cancer, high level CIN and control groups.

\begin{tabular}{lrrrcc}
\hline Group & Case & Type I & Type II & Type III & Type IV \\
\hline Cervical cancer & 6 & 0 & 0 & 2 & 4 \\
High level CIN & 104 & 16 & 74 & 14 & 0 \\
Cervicitis & 162 & 132 & 28 & 2 & 0 \\
\hline
\end{tabular}

CIN, cervical intraepithelial neoplasia.

difference on blood flow of cervix between chronic cervicitis and CINI was not significant. Cervical blood flow was not rich and blood flow signals were few. Type I dominated, accounting for $81.48 \%$ constituted the high level CIN group, in which blood flow was relatively rich in some regions of the cervix, and strip or 'short rod-like' vessels were evident. Type II dominated, accounting for $71.15 \%$ constituted the early stage cervical carcinoma group, in which cervical blood flow was rich, mussy, and distorted, in the form of fire balls. Type III dominated and type IV accounted for 66.67\% (Figs. 1-3; Table III).

\section{Discussion}

Clinical samples have shown that the 5-year survival rate of patients at the early stage of cervical cancer may reach up to $80-90 \%$ if they diagnosed and treated in a timely manner $(1,2)$. Therefore, a timely and effective screening of precancerous lesions and early stage cervical cancer has become the key for preventing and treating cervical cancer and reducing mortality (5). Present cervical cancer screening methods used mainly include exfoliate cytology examination (Pap smear, Thinprep Cytologic Test, TCT), HPV detection (HCII, HR-HPV typing), and cytology and HPV detection (6). These are convenient procedures involving simple operation, high smear collection and a high detection rate of abnormal cervical cells, which have made TCT the most important method for the screening of cervical lesions, which plays a significant role in the screening of cervical cancer $(5,6)$. However, TCT detection is greatly affected by subjective factors. For the same smear, different cytological experts may have different film reading results. It may be because diagnosis was made on the basis of cytological morphological characteristics, lacking the assessment of histological characteristics, thus increasing the rate of misdiagnosis. Normally, the sensitivity of TCT detection is $55-80 \%$. Once abnormal cells are discovered, its specificity reaches $90 \%$. We showed that TCT has sensitivity of 53.13\%, specificity of $79.01 \%$, and a pathological coincidence rate of $82.93 \%$, which again proved that TCT had better specificity.

At present, it has been acknowledged that persistent high-risk HPV infection is the fundamental pathogenic factor for cervical cancer (7). Thus, HPV is important in the early detection, treatment and follow-up of cervical lesions. Recent literature has shown that single HPV detection is the essential condition rather than the sufficient condition for cervical cancer (7). HPV inspection has a high sensitivity and poor specificity. Our study results showed that when HPV screening was performed independently, its sensitivity was $90.63 \%$, 


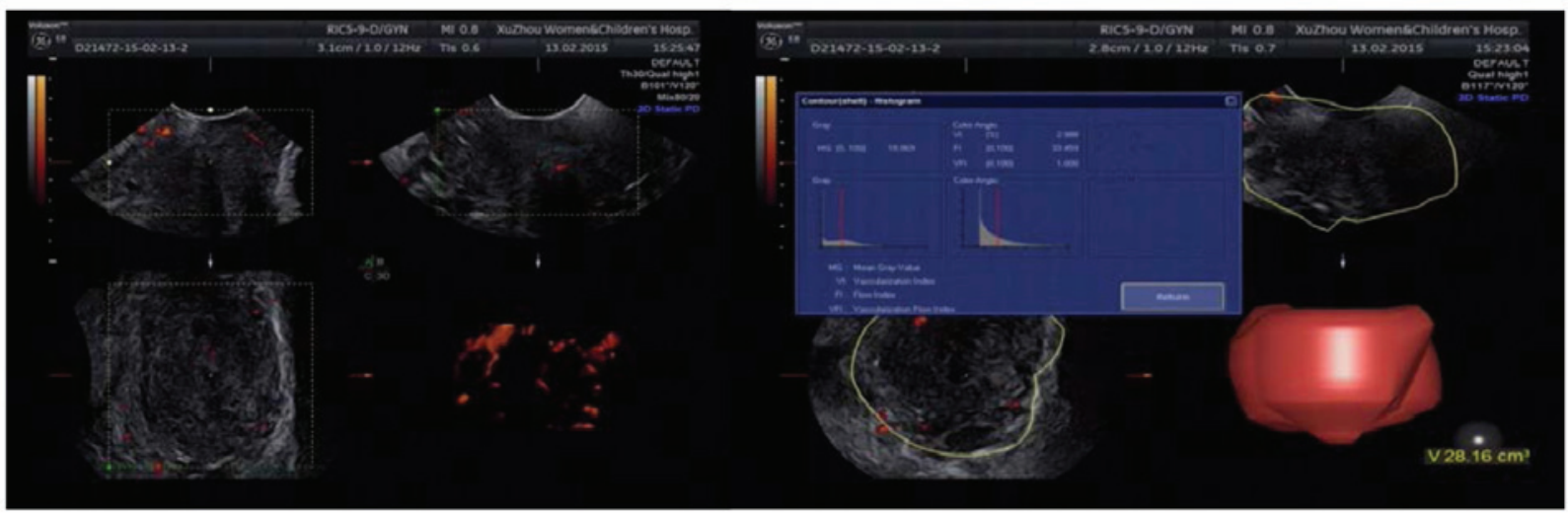

Figure 1. Cervicitis, blood flow of the cervix was not rich and blood flow signals were quite few. Type I dominated.

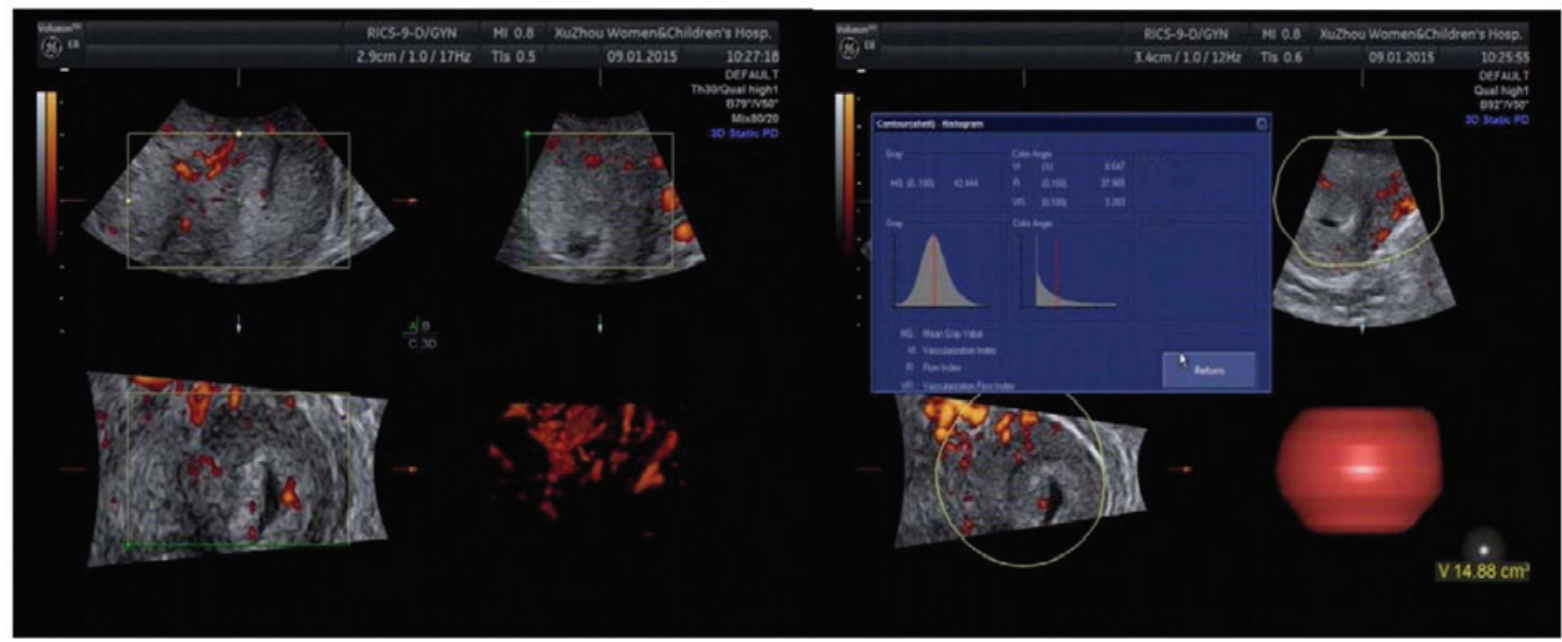

Figure 2. High level CIN, blood flow in some regions was quite rich, strip or 'short rod-like' vessels could be seen. Type II dominated. CIN, cervical intraepithelial neoplasia.

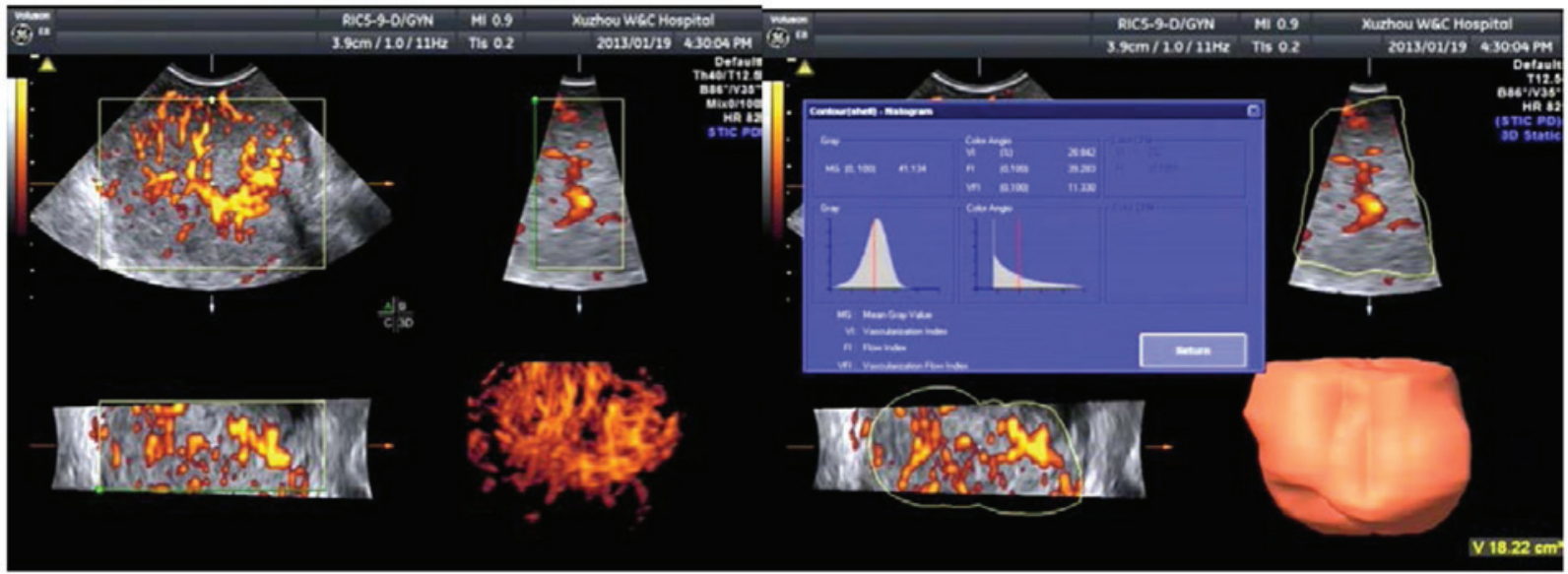

Figure 3. Cervical cancer, the volume of the cervix was enlarged and even out of normal shape, and the branches of vessels were complicated, distorted and irregular, nonuniform, distortedly clustered like fire balls. Type III-IV dominated.

specificity was $72.84 \%$, and the diagnostic coincidence rate was $83.73 \%$, whereas the diagnostic coincidence rate with high level CIN was only $37.68 \%$. Since most HPV-positive patients may become HPV-negative and the lesions would not develop into cervical lesions, such a result would undoubtedly aggravate seeking medical assistance and lead to a series of 
unnecessary examinations and treatments. HPV detection is considered significant for condensing the high-risk patients, distributing the ASCUS and LSIL of undetermined significance, and monitoring the precancerous lesions as well as the conditions following treatment (8-10). Sensitivity of HPV detection is extremely high; however, the false-positive rate is also extremely high. It may be just a virus infection, and not necessarily the results of cervical cytological and pathological changes. Thus, a safe and effective joint detection method for early diagnosis of cervical cancer should be identified.

Our data showed that HPV infection was 16, 58, 52, 33, 31 and 18 from high to low. HPV16(+) accounted for $83.33 \%$ $(5 / 6)$ in the cervical cancer group, for $56.12 \%(55 / 98)$ in the high level CIN group, and for 25\% (32/128) in the CINI group. These results further confirmed that HPV16 had the highest carcinogenicity, thus requiring the most serious screening and management. HPV18 was rarely seen in our data.

Previous studies have confirmed from the molecular level that angiogenesis is important in the occurrence and development of cervical cancer, and suggested treating angiogenesis as an independent factor for the diagnosis of cervical cancer $(6,8)$. Microvessel density (MVD) may reflect angiogenesis activity (11). It has been used as the gold standard to assess the state of angiogenesis. However, the blood vessel density measured by immunohistochemistry is changeable, unable to provide an overall blood supply information of tumor, or guide the decision of therapeutic regimen (12). Thus, a more favorable proxy indicator that is safe, non-invasive, and well-correlated with MVD and that could express the vascular condition of tumor should be identified. 3D-CPA technology is newly developed and has combined power of Doppler mode and the $3 \mathrm{D}$ volume sampling mode. It was able to reveal the vascular structures inside and around the tumor in a dynamic, 3D and all-round manner as well as the vascular trees inside the tumor in an intuitive and complete manner. Being characterized for relative independence, high sensitivity and repeatability, 3D-CPA technology could detect the direction, distribution, and shape of low blood perfusion of micro-vessels inside the tumor, thus reflecting the blood supply condition of tumor. By means of VOCAL software, 3D-CPA supports quantitative analysis on the vessels as well as blood flow inside the tumor, which is conducive to a qualitative diagnosis and treatment effect evaluation of the tumors (9). 3D vascular parameters computed from VOCAL includes VI, flow index (FI) and vascularization flow index (VFI). VI was used to measure the number of color information in the region of interest and show the number of vessels detected inside tissues. As for the credibility of VI in assisting cervical lesion detection, some literature has reported that 3D color power Doppler's vascular tying and VI were correlated with the expression of MVD and VEGF, which indicated that vascular typing and VI may reflect the vascular expression of the tumor (10). Besides, the results of our previous study also showed that, VI and VFI of the cervical cancer group were significantly higher than those of the CIN and cervicitis groups, and the difference was statistically significant $(\mathrm{P}<0.05)$. Such a result confirmed that $3 \mathrm{D}$ color power Doppler ultrasonography may reflect the blood supply state of cervix objectively, providing important reference for the ultrasonic diagnosis of early cervical cancer and CIN. Thus, it could be confirmed that 3D color power Doppler ultrasonography was of great application value (13). In the present study, we performed a screening independently by VI and obtained the following parameters: sensitivity, $73.44 \%$; specificity, 83.95\%; and diagnostic coincidence rate, $77.51 \%$. Although sensitivity was relatively lower than HPV, its specificity was higher. Notably, it was non-invasive, safe and had very good repeatability.

A semi-quantitative analysis on the vessels and blood flow in the area of cervical lesion of the early cervical cancer, high level CIN, low level CIN and cervicitis groups was performed. The results showed (Table II) that the typing of cervical blood flow in the former three groups were significantly different. The cervical cancer group had rich blood flow, the volume of the cervix was enlarged and even out of normal shape, the branches of vessels were complicated, distorted and irregular, non-uniform, distortedly clustered in the form of fire balls and was dominated by type III-IV, of which type IV accounted for $66.67 \%$. The blood flow in the chronic cervicitis and low level CIN groups had no significant difference, indicating that blood flow change in the early period of disease was not as obvious. Thus, it was difficult to identify the mild lesion purely through ultrasonic vessel typing; the cervical blood flow of the cervicitis group was not rich and blood flow signals were few, with type I dominated, accounting for $81.48 \%$; blood flow in some regions of the high level CIN group was quite rich, and strip or 'short rod-like' vessels were evident, with type II dominated, accounting for $71.15 \%$, indicating that cervix had new vessels. Based on the above analysis, we concluded that cervical vessels could reflect the degree of cervical lesions more objectively, which combined with VI, could provide more reliable evidence for the diagnosis and identification of the high level CIN and early cervical cancer.

The American Cancer Society (ACS), American Society of Colposcopy and Cervical Pathology (ASCCP) and American Society for Clinical Pathology (ASCP) contended that the optimal strategy for cervical cancer screening is to maximize the benefits of screening while minimizing the potential hazards (14). The most commonly used methods for cervical screening at present are TCT and HPV. The sensitivity of TCT is low and extremely subjective while the specificity of HPV is relatively low. To make the early diagnosis of cervical cancer more accurate, reliable and predictive, there is a need for a reliable index or method to assist the above detection methods for more comprehensive judgment. In the present study, we have compared the sensitivity, specificity, diagnostic rate, and Youden index of individual 3D-CPA, HR-HPV, and TCT screening, HR-HPV+TCT screening, 3D-CPA+HR-HPV+TCT screening on cervical lesions. The results of the present study show that the sensitivity of 3D-CPA+HR-HPV+TCT screening reached $94.53 \%$, and although its specificity was relatively lower than that of the individual VI screening, it was still higher than that of HR-HPV+TCT screening. Additionally, its diagnostic coincidence rate reached $89.47 \%$, which was the highest rate, while the Youden index was also the highest, reaching up to 0.760 . These results indicated that $3 \mathrm{D}-\mathrm{CPA}+\mathrm{HR}-\mathrm{HPV}+\mathrm{TCT}$ screening had the best accuracy. Combined with the differences of cervical high-level lesion and cervical cancer cervical blood flow typing, the sensitivity of screening was further improved, which indicated that 3D-CPAHR-HPV+TCT screening had extremely high clinical application value. 
In conclusion, by comparing the correlation between 3D-CPA, HR-HPV, TCT detection and clinical pathological outcomes, we concluded that th ejoint detection of the three methods may significantly improve the disease detection rate and screening authenticity. The 3D-CPA technology can be used as an auxiliary method for cervical cancer screening. However, since different groups have different characteristics, the medical environment and resources should be considered. Thus, the most effective screening method for patients according to their clinical performances, high-risk factors of lesion and personal economic conditions should be identified, for improved contributions to the clinic.

\section{References}

1. Castellsagué X, de Sanjosé S, Aguado T, Louie, L. Bruni KS, Muñoz J, Diaz M, Irwin K, Gacic M, Beauvais O, et al: HPV and Cervical Cancer in the World 2007 Report. Vol. 25, 2007 (http:// www.hpvcentre.net/link_media/HPVReport2007.pdf.)

2. PAHO publication: Estrategia y Plan de Acción Regionales sobre la Prevención y el Control del Cáncer Cervicouterino. Washington DC, 2008 (www.rho.org/files/PAHO_Regional_ Strategy_2010_sp.pdf.) (In Spanish).

3. Sherman SM, Moss E and Redman CW: The invasive cervical cancer review: Psychological issues surrounding disclosure. Cytopathol 24: 77-80, 2013.

4. Jiao G, Xu H and Ren L: The diagnostic value of three-dimensional ultrasound in invasive cervical tuberculosis. J Tongji Univ 31: 85-87, 2010. (Medical edition).

5. Johnston EI and Logani S: Cytologic diagnosis of atypical squamous cells of undetermined significance in perimenopausal and postmenopausal women: lessons learned from human Papillomavirus DNA testing. Cancer 111: 160-165, 2007.
6. Tjalma W, Van Marck E, Weyler J, Dirix L, Van Daele A, Goovaerts G, Albertyn G and van Dam P: Quantification and prognostic relevance of angiogenic parameters in invasive cervical cancer. Br J Cancer 78: 170-174, 1998.

7. Meijer CJ, Snijders PJ and van den Brule AJ: Screening for cervical cancer: Should we test for infection with high-risk HPV? CMAJ 163: 535-538, 2000.

8. Landt S, Mordelt K, Schwidde I, Barinoff J, Korlach S, Stöblen F, Lichtenegger W, Sehouli J and Kümmel S: Prognostic significance of the angiogenic factors angiogenin, endoglin and endostatin in cervical cancer. Anticancer Res 31: 2651-2655, 2011.

9. Pairleitner H, Steiner H, Hasenoehrl G and Staudach A: Three-dimensional power Doppler sonography: imaging and quantifying blood flow and vascularization. Ultrasound Obstet Gynecol 14: 139-143, 1999.

10. Li P, Wang X and Zhang Y: Correlation between the blood flow of cervical cancer and MVD, VEGF under three-dimensional energy doppler ultrasound detection. J China Med Univ 38: 846, 2009.

11. Weidner N, Semple JP, Welch WR and Folkman J: Tumor angiogenesis and metastases correlation in invasive breast carcinoma. N Engl J Med 324: 1-8, 1991.

12. Hsu KF, Su JM, Huang SC, Cheng YM, Kang CY, Shen MR, Chang FM and Chou CY: Three-dimensional power Doppler imaging of early-stage cervical cancer. Ultrasound Obstet Gynecol 24: 664-671, 2004.

13. Liang H, Fu-Min L, Lei S, Peng L and Jian Z: Transvaginal three-dimensional color power Doppler ultrasound and cervical MVD measurement in the detection of cervical intraepithelial neoplasia. Eur Rev Med Pharmacol Sci 18: 1979-1984, 2014.

14. Saslow D, Castle PE, Cox JT, Davey DD, Einstein MH, Ferris DG, Goldie SJ, Harper DM, Kinney W, Moscicki AB, et al: American Cancer Society Guideline for human papillomavirus (HPV) vaccine use to prevent cervical cancer and its precursors. CA Cancer J Clin 57: 7-28, 2007. 